\title{
Influence of K/ Na Component Ratio on Physical Properties of KNN Ceramics
}

\author{
Le Tran Uyen Tu*, Nguyen Thi Tuyet Trinh, Dung Thi Hoai Trang, Vo Thanh Tung* \\ Physics faculty, University of Sciences, Hue University \\ Hue City, Vietnam
}

\begin{abstract}
In recent years, considerable efforts have been made toward the control of processing techniques, volatile mass and component ratio of Potassium and Sodium in order to improve physical properties of KNN ceramics. In this paper, the influence of physical properties of the $\left(\mathrm{K}_{x} \mathrm{Na}_{1-x}\right) \mathrm{NbO}_{3}$ ceramics, fabricated using conventional solid state reaction method, on $\mathrm{K} /$ $\mathrm{Na}$ component ratio was examined. The densities of ceramic sample changed depending on the variation of $x$ parameter from 0.47 to $0.52 \mathrm{~mol} \%$. The highest value of density is $4.26 \mathrm{~g} / \mathrm{cm}^{3}$ at potassium content $x$ of $0.48 \mathrm{~mol} \%$, coresponding with the uniform in microstructure of ceramic surface. Effects of $\mathrm{K} / \mathrm{Na}$ ratios in the vicinity of morphotropic phase boundary on microstructure, ferroelectric and piezoelctric properties are also examined. The $\left(\mathrm{K}_{0.48} \mathrm{Na}_{0.52}\right) \mathrm{NbO}_{3}$ system sintered at $1090{ }^{\circ} \mathrm{C}$ shows the highest values of remanent polarization $P_{r}=3,19$ $\mu \mathrm{C} / \mathrm{cm}^{2}$ at the applied coercive electric field $E_{c}=7,16 \mathrm{kV} / \mathrm{cm}$. At $\mathrm{x}=0.48 \mathrm{~mol} \%$, electromechanical coupling factor $k_{p}$ of 0.26 and the piezoelectric constant $d_{33}$ of $69 \mathrm{pC} / \mathrm{N}$ also can be obtained, related to homogenous grains size of orthohombic phase..
\end{abstract}

Keywords: Ratio of $\mathrm{K} / \mathrm{Na}$, piezoelectric, KNN-based lead-free ceramics.

\section{INTRODUCTION}

In previous decades, piezoelectric ceramic systems basing on titanium zirconate lead (PZT) are widely studied and applied in many fields[1-3]. However, due to the toxicity of lead $[4,5]$, the development of lead-free piezoelectric ceramic systems with exellent electric and piezoelectric properties to replace lead based ceramics in different devices is necessary [6].

Among lead-free systems, ceramics based on $(\mathrm{Na}, \mathrm{K}) \mathrm{NbO}_{3}$ (KNN) have attracted a lot of attention due to their prominent ferroelectric properties, high Curie temperature (about $420{ }^{\circ} \mathrm{C}$ ) and environmentally friendly, capable of replacing the lead based ceramics. However, it is difficult to manufacture KNN ceramics with a high density and good electrical properties using conventional sintering process due to the high volatility of alkaline elements at high sintering temperature [7, 8]. In additional, the ratio of $\mathrm{K} / \mathrm{Na}$ in the vicinity of morphotropic phase boundary (MPB) is also one of the main reasons affecting the properties of KNN ceramics. Some reports suggested that the KNN ceramic with the ratio of $\mathrm{K} / \mathrm{Na}$ components at $0.5 / 0.5$ has exellent dielectric and piezoelectric properties due to the existence of morphological phase boundary between two phases [9, 10]. Meanwhile, other reports issued physical properties of the KNN system are dominated by presence of not morphotropic phase boundary but polymorphic phase boundary $[11,12]$. In addition, the exact $\mathrm{K} / \mathrm{Na}$ component ratio to obtain $\mathrm{KNN}$ ceramics with prominent characteristics is sensitive with raw compositions. That is, the effect of $\mathrm{K} / \mathrm{Na}$ ratio on the physical properties of KNN ceramics has been still controversial.

In this paper, ceramics with the chemical formula $\left(\mathrm{K}_{x} \mathrm{Na}_{1-}\right.$ $\left.{ }_{x}\right) \mathrm{NbO}_{3}$ are made using conventional solid state reaction methods. The changes in structural characteristics, dielectric, ferroelectric and piezoelectric properties of fabricated ceramics when potassium component $(x)$ varies from 0.47 to $0.52 \mathrm{~mol} \%$ has been carried out. Results of this paper hope to contribute the understanding about microstructure and physical properties of KNN ceramics depending on the change of ratio potassium/ sodium component.

\section{EXPERIMEMTAL}

The general formula of the studied material was $\left(\mathrm{K}_{x} \mathrm{Na}_{1}\right.$ $\left.{ }_{x}\right) \mathrm{NbO}_{3}$, where $x$ are $0.47,0.48,0.49,0.50$, and $0.52 \mathrm{~mol} \%$; respectively. Reagent grade oxide powders (purity $\geq 99 \%$ ) of $\mathrm{K}_{2} \mathrm{CO}_{3}, \mathrm{Na}_{2} \mathrm{CO}_{3}, \mathrm{Nb}_{2} \mathrm{O}_{5}$ were used as raw materials. Before being weighed, the $\mathrm{K}_{2} \mathrm{CO}_{3}$ and $\mathrm{Na}_{2} \mathrm{CO}_{3}$ powders were dried in an oven at $200{ }^{\circ} \mathrm{C}$ for $2 \mathrm{~h}$ to minimize the effect of moisture. Mixed powder was milled in 8 hours to obtain an appropriate distribution of the particle size, the small particle size will improve the reactivity of the powders [13]. Two calcinations at temperature $850{ }^{\circ} \mathrm{C}$ for $2 \mathrm{~h}$ were then performed to obtain compositionally homogeneous powders and the single phase formation [14]. Thereafter the calcined, powders were ball milled again for $16 \mathrm{~h}$ to create a more uniform distribution of powders and the reaction occurs completely [14]. The ground materials were pressed into disk $12 \mathrm{~mm}$ in diameter and 1.5 $\mathrm{mm}$ in thick under $100 \mathrm{MPa}$. In order to limit the evaporation of alkaline elements, these pellets were covered by the powders with the same composition and then were sintered in a sealed alumina crucible at the temperature of $1090{ }^{\circ} \mathrm{C}$ for 2 $\mathrm{h}$

The crystal structures of the sintered samples were examined by $\mathrm{X}$-ray diffraction with $\mathrm{CuK} \alpha$ radiation, $\lambda=$ $0.15406 \mathrm{~nm}$ (XRD, D8 ADVANCE-Bruker). The grain morphology of the samples was examined by scanning electron microscopy (Novanano SEM 450-Fei). The densities of samples were measured by Archimedes method. The ceramic samples were poled in a silicone oil bath at $80{ }^{\circ} \mathrm{C}$ by applying dc field of $30 \mathrm{kV} / \mathrm{cm}$ for 20 min then cooling under the same electric field. They were aged for $24 \mathrm{~h}$ prior to testing. 
The dielectric constant at room temperature is calculated by measuring the capacitance $\mathrm{C}$ of samples using RLC HIOKI 3532 at frequency of $1 \mathrm{kHz}$. The ferroelectric property was measured by Sawyer-Tower method. The piezoelectric properties were determined from the resonance and antiresonance frequency by using an Impedance Analyzer HP 1493A and RLC HIOKI 3532.

\section{RESULTS AND DISCUSSION}

Fig. 1(a) shows the density of the $\left(\mathrm{K}_{x} \mathrm{Na}_{1-x}\right) \mathrm{NbO}_{3}$ ceramic samples sintered at $1090{ }^{\circ} \mathrm{C}$ as a function of the Potassium (K) content. The $\left(\mathrm{K}_{0.47} \mathrm{Na}_{0.53}\right) \mathrm{NbO}_{3}$ ceramics shows low density, $4.08 \mathrm{~g} / \mathrm{cm}^{3}$. With increasing of $\mathrm{K}$ content, the density initially increases and reaches the maximum value of $4.26 \mathrm{~g} / \mathrm{cm}^{3}$ at $0.48 \mathrm{~mol} \%$ Potassium, then decreases. These results are consistent with microstructure of fracture surfaces of the samples as shown in Fig.1(b)-1(f). As shown in Fig. 1(b), the microstructure of the $\left(\mathrm{K}_{0.47} \mathrm{Na}_{0.53}\right) \mathrm{NbO}_{3}$ consisted of irregular large and small grains, the discrete distributions, porous. However, the microstructure of samples becomes denser and more uniform as the Potassium content increases from 0.47 to $0.48 \mathrm{~mol} \mathrm{\%}$ (Fig. 1(c)).
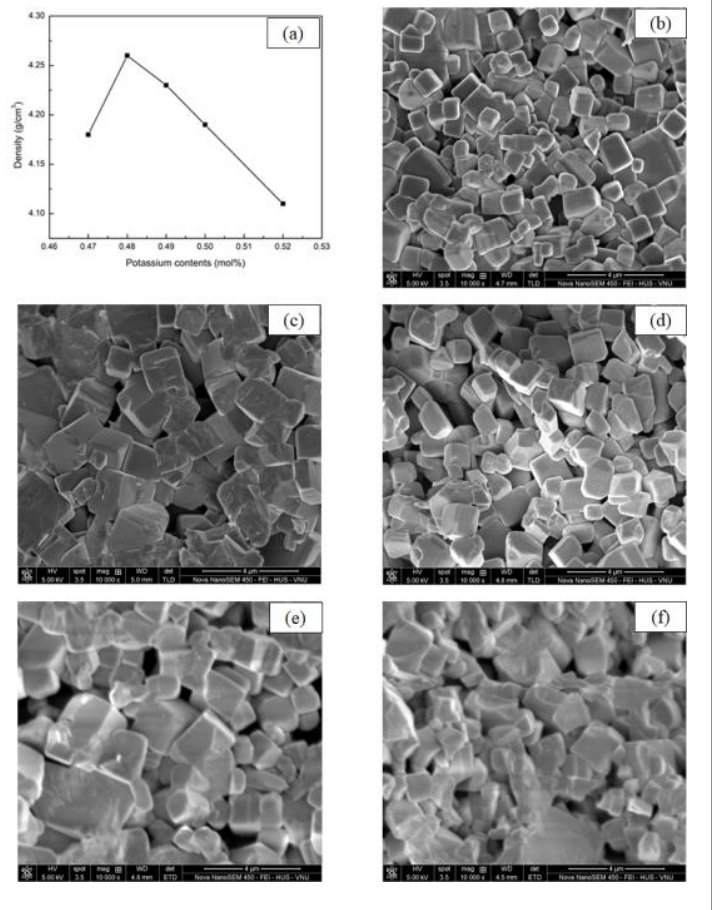

Fig. 1. The Potassium content dependence of the density of KNN ceramics measured at room temperature and the SEM micrographs of $\left(K_{x} N a_{I_{-x}}\right) \mathrm{NbO}$ ceramics with $x=0.47(b), x=0.48(c), x=0.49(d), x=0.50(e)$ and $x=0.52(f)$.

Fig. 1(d), (e) and (f) show that with further increasing Potassium content to $0.52 \mathrm{~mol} \%$, the particle size increased sharply. In addition, the shape and size of particles were no onger uniform. Such with the $0.48 \mathrm{~mol} \%$ Potassium, homogeneous microstructure of ceramic was obtained.

Fig. 2 shows the XRD patterns at room temperature with the $2 \theta$ range from $20^{\circ}$ to $80^{\circ}$ of $\left(\mathrm{K}_{x} \mathrm{Na}_{1-x}\right) \mathrm{NbO}_{3}$ ceramic samples sintered at $1090{ }^{\circ} \mathrm{C}$. It can be seen that all samples with $x$ from 0.47 to $0.52(\mathrm{~mol} \%)$ ) exhibited single phase structure of perovskite $\mathrm{ABO}_{3}$, without trace of secondary phases. In order to distinguish whether the fabricated samples have orthorhombic or tetragonal structure, the intensities of the

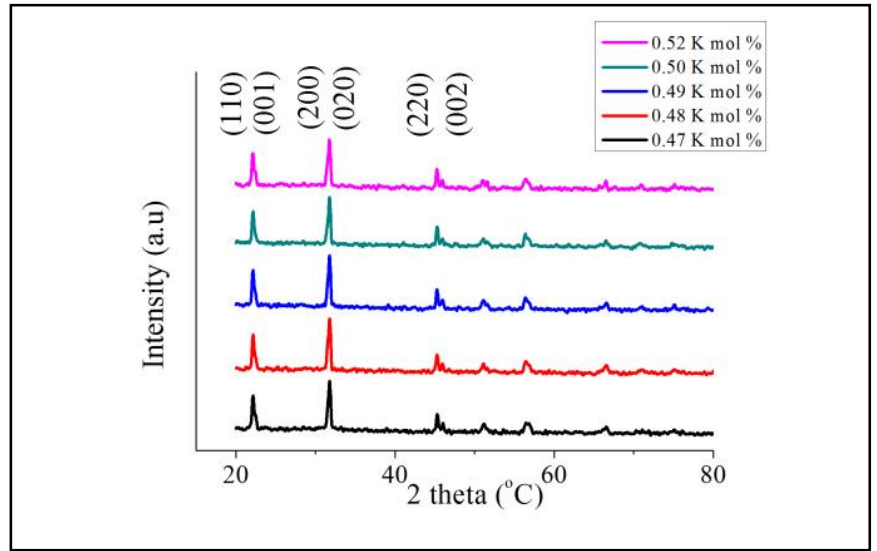

Fig. 2. $\quad X$ ray diffraction patterns of the $\left(\mathrm{K}_{x} \mathrm{Na}_{1-x}\right) \mathrm{NbO}_{3}$ ceramics

double peaks in the $\mathrm{X}$-ray diffraction patterns are further considered. The corresponding XRD patterns characterized by the double peaks in which intensity of (110) and (220) peaks higher than that of (001) and (020) peaks respectively. According to Skidmore and co.authors report, this indicated that all ceramic samples have orthorhombic phase [15]. In addition, the intensity of the double diffraction peaks in Fig. 2 is the same through all $\mathrm{x}$ values. This result shows that the phase structure of $\mathrm{KNN}$ system is completely unaffected by the changes of $\mathrm{K} / \mathrm{Na}$ component ratio in the range $x=$ $(0.47 \div 0.52) \mathrm{mol} \%$. The changes of potassium content also significantly affect electric properties of $\left(\mathrm{K}_{x} \mathrm{Na}_{1-x}\right) \mathrm{NbO}_{3}$ ceramics.

Fig. 3 shows the room temperature dielectric constant $\varepsilon$ and dielectric loss $\tan \theta$ measured at $1 \mathrm{kHz}$ frequency of $\left(\mathrm{K}_{x} \mathrm{Na}_{1-x}\right) \mathrm{NbO}_{3}$ ceramics as a function of the potassium contents $(x)$. The dielectric constant $\varepsilon$ increases with the rise in potassium content and reaches value (391) at $x=0.48$. However, when $x \geq 0.49$, the dielectric constant $\varepsilon$ decreases. This is related to density and microstructure of ceramics. The varying tendency of dielectric loss tangent with the potassium contents is inverse to that of the measured dielectric constant. The minimum value of dielectric loss obtained at $x=0.48 \mathrm{~mol}$ $\%$ potassium is 0.07 .

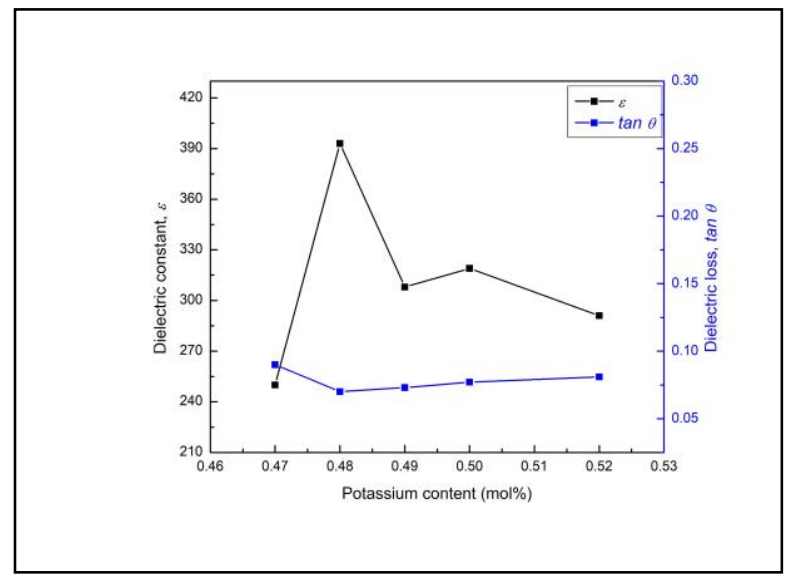

Fig. 3. Room-temperature dielectric constant $\varepsilon$ and dielectric loss tan $\theta$ of KNN ceramics as a function of the potassium ( $x$ mol \%) content 


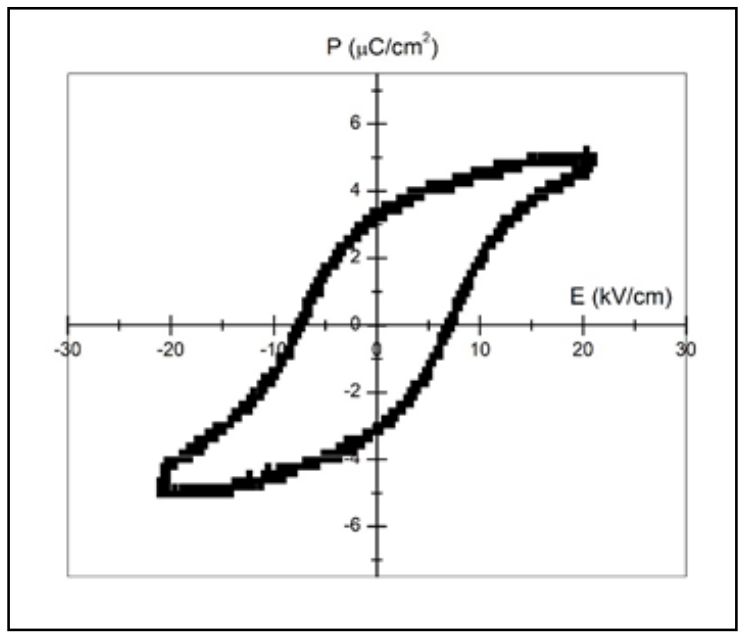

Fig. 4. Hysteresis loops of $\left(\mathrm{K}_{0.48} \mathrm{Na}_{0.52}\right) \mathrm{NbO}_{3}$ ceramic sample measured at room temperature

Fig. 4 shows the shape of ferroelectric hysteresis loops of $\left(\mathrm{K}_{0.48} \mathrm{Na}_{0.52}\right) \mathrm{NbO}_{3}$ sample measured at room temperature. Then, the remanent polarization $P_{r}$ and the coercive field $E_{C}$ of ceramics as a function of the potassium contents $(x)$ were determined, as shown in Fig. 5. Experimental results show that pure KNN ceramics has a typical hysteresis loop of ferroelectric materials. With increasing of potassium component $(x \% \mathrm{~mol})$, initially the remanent polarization tends to increase, reaches the maximum value of $P_{r}=3.19 \mu \mathrm{C} / \mathrm{cm}^{2}$ at $x=0.48 \mathrm{~mol} \%$, and then deacreases. Meanwhile, the coercive field decreased to the lowest value of $E c=7.26 \mathrm{kV} /$ $\mathrm{cm}$ as the same potassium content, after that raises again.

To determine piezoelectric properties of $\left(\mathrm{K}_{x} \mathrm{Na}_{1-x}\right) \mathrm{NbO}_{3}$ ceramics sitering at $1090{ }^{\circ} \mathrm{C}$, resonant vibration spectra of samples were measured at room temperature. From these resonant spectra, piezoelectric parameters of samples were obtained.

Fig. 6 shows the electromechanical coupling factors of radial vibration mode $\left(k_{p}\right)$ and piezoelectric constant $\left(d_{33}\right)$ changing as a function of the potassium content. At $0.48 \mathrm{~mol} \%$ of potassium, the electrical parameters became prominent due to the well-arrangement of microstructure of the ceramics.

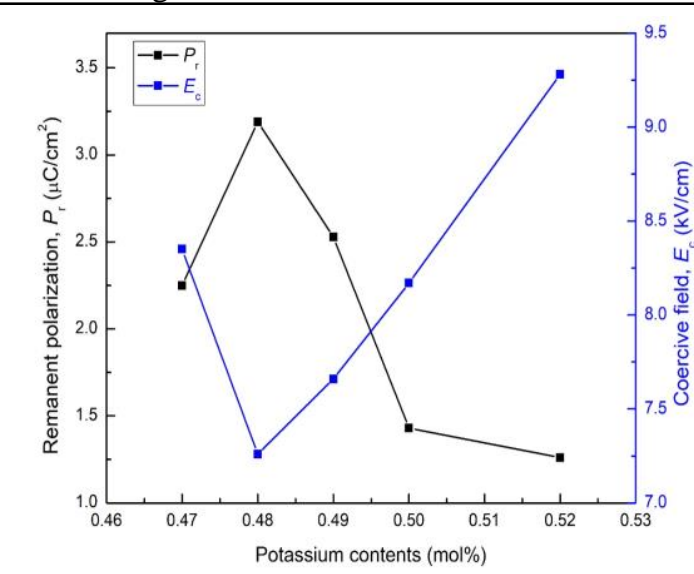

Fig. 5. The remnant polarization $\left(P_{r}\right)$ and coercive field $\left(E_{c}\right)$ of $\left(K_{x} N a_{1-x}\right) N_{b} O_{3}$ ceramics as a function of the potassium ( $x \mathrm{~mol} \%$ ) content

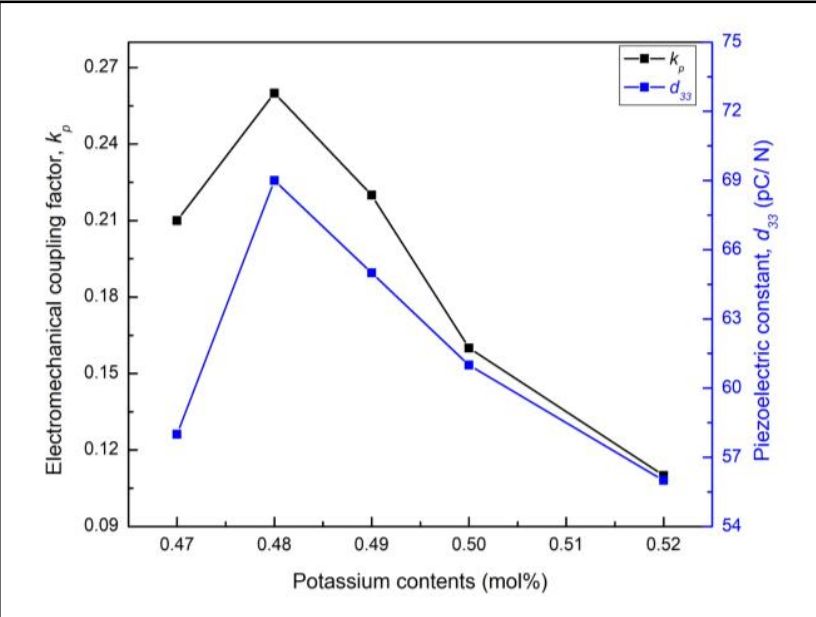

Fig. 6. The potassium content dependence of the values $k_{p}$ and $d_{33}$ of $\left(K_{x} N a_{1}\right.$ $\left.{ }_{x}\right) \mathrm{NbO}_{3}$ ceramics

The largest values for $k_{p}$ of $0.26, d_{33}$ of $69 \mathrm{pC} / \mathrm{N}$, reached at $x=0.48 \mathrm{~mol} \%$. And then piezoelectric parameters rapidly decrease when potassium content continue raising. These properties of $\left(\mathrm{K}_{x} \mathrm{Na}_{1-x}\right) \mathrm{NbO}_{3}$ ceramics at $x$ of $0.48 \mathrm{~mol} \%$ corresponding the microstructures of well-faceted and homogeneously distributed grains.

\section{CONCLUSION}

The lead-free KNN ceramics with different $\mathrm{K} / \mathrm{Na}$ ratios in the vicinity of morphotropic phase boundary were fabricated. All samples has simple perovskite structure. The effect of ratios of $\mathrm{K} / \mathrm{Na}$ component on the structure, microstructure and dielectric, ferroelectric properties of $\left(\mathrm{K}_{x} \mathrm{Na}_{1-x}\right) \mathrm{NbO}_{3}$ ceramics was studied. The KNN ceramic with $x=0.48 \mathrm{~mol} \%$ sintered at $1090{ }^{\circ} \mathrm{C}$ shows smallest values of dielectric loss and coercive field. The best dielectric constant, remanent polarization, electromechanical coupling factors, and piezoelectric constant were obtain at potassium component content of $0.48 \mathrm{~mol} \%$ of KNN ceramic, corresponding to the highest density and homogenous grains size.

\section{REFERENCES}

[1] K. Uchino, Piezoelectric Actuators and Ultrasonic Motors.Kluwer Academic Publishers, Boston, (1997).

[2] B. Jaffe, W. R. Cook, and H. Jaffe (Eds.), Piezoelectric Ceramics, Academic, New York (1971).

[3] Y. Xu (Eds.), "Ferroelectric Materials and Their Applications", Elsevier Science, Amsterdam-London-New York-Tokyo (1991).

[4] Saito Y, Takao H, Tani T, Nonoyama T, Takatori K, Homma, Nagoya, T, Nakamura M., "Lead-free piezoceramics",Nature 432, 84-87 (2004).

[5] In-Young Kang, In-Tae Seo, Yu-Joung Cha, Jae-Hong Choi, Sahn Nahm,Tae-Hyun Sung, Jong-Hoo Paik, "Low temperature sintering of $\mathrm{ZnO}$ and $\mathrm{MnO}_{2}$-added $\left(\mathrm{Na}_{0.5} \mathrm{~K}_{0.5}\right) \mathrm{NbO}_{3}$ ceramics". Journal of the European Ceramic Society 32, 2381-2387 (2012)

[6] EU-Directive 2002/96/EC, "Waste Electrical and Electronic Equipment (WEEE)," Off. J. Eur. Union, 46 [L37] 24-38 (2003).

[7] Jing-Feng Li, Ke Wang, Fang-Yuan Zhu, Li-Qian Cheng, and FangZhou Yao, "(K,Na) $\mathrm{NbO}_{3}$-Based Lead-Free Piezoceramics": Fundamental Aspects, Processing Technologies, and Remaining Challenges, J. Am. Ceram. Soc.,96 3677-3696 (2013).

[8] Wenxin Ma, Xinghua Fu1, Wenhong Tao, Lei Yang, Guoyuan Cheng and Liping Zhao, "KNN-Sb lead-free piezoelectric ceramics synthesized by hydrothermal method", Materials Science Forum, Vol 859, pp 3-7 (2016).

[9] V. J. Tennery and K. W. Hang, "Thermal and X-Ray Diffraction Studies of $\mathrm{NaNbO}_{3}-\mathrm{KNbO}_{3}$ System”, J. Appl. Phys., 39 [10] 4749-53 (1968). 
[10] Y. J. Dai, X. W. Zhang, and K. P. Chen, "Morphotropic Phase Boundary and Electrical Properties of $\mathrm{K}_{1 \_\mathrm{x}} \mathrm{Na}_{\mathrm{x}} \mathrm{NbO}_{3}$ Lead-Free Ceramics", Appl. Phys. Lett., 94 [4] 042905, 3pp (2009).

[11] Y. Dai, X. Zhang, G. Zhou, Phys. Lett, 90 (2007).

[12] F.-Z. Yao, K. Wang, J.-F. Li, J. Appl. Phys, 113, 174105 (2013)

[13] F. Rubio-Marcos, J. J. Romero, M. G. Navarro-Rojero, and J. F. Fernandez, "Effect of $\mathrm{ZnO}$ on the Structure, Microstructure and Electrical Properties of KNN-Modified Piezoceramics", J. Eur. Ceram. Soc., 29, 3045-52 (2009).
[14] Dai Y., Zhang X. and Zhu G., "Phase transitional behavior in $\mathrm{K}_{0.5} \mathrm{Na}_{0.5} \mathrm{NbO}_{3}-\mathrm{LiTaO}_{3}$ ceramics”, Appl. Phys. Lett., 90, 262903 (2007).

[15] T.A. Skidmore, T. Stevenson, T.P. Comyn, S.J. Milne "Phase Development During Mixed-Oxide Processing of a $\left[\mathrm{Na}_{0.5} \mathrm{~K}_{0.5} \mathrm{NbO}_{3}\right] 1-\mathrm{X}$ - $\left[\mathrm{LiTaO}_{3}\right] \mathrm{x}$ Powder", Key Engineering Materials, 368-372(HighPerformance Ceramics V), 1886-9 (2008). 